\title{
The Design of the Online Teaching System based on Multi-agent System ${ }^{1}$
}

\author{
Ablimit Arxiden \\ Faculty of Computer and Information Science, \\ Southwest University, Chongqing 400715, China \\ Faculty of Mathematics and Information, Teachers \\ Collage of Hotan 848000 \\ *Corresponding author: abtht@163.com
}

\author{
Shanxia Wang \\ Faculty of Computer and Information Science, \\ Southwest University, \\ Chongqing 400715, China
}

\begin{abstract}
By introducing multi-agent technology to the online teaching system and establishing a personalized online teaching system model, the intelligent and personalized problems in the traditional online teaching system are solved; the needs of students for learning are fulfilled; the intelligent and interactive functions of the online teaching system are improved; a new interactive online teaching model is implemented. In this paper, the online system design based on multi-agent system technology is raised, and the modules and functions of the system are defined.
\end{abstract}

Keywords- Multi-agent System; Intelligent Model; Online Teaching System

\section{Introduction}

In the teaching process, the teaching system based on the Internet arises constantly. On the basis of analyzing the differences between online teaching and traditional teaching, the basic structure of a multi-agent technology based online teaching system including website presenting teaching contents, complete course management system, virtual classroom environment, diversified interactive mechanism and fully-featured teaching test system is constructed and expounded, so as to provide a reference for people to develop an online teaching system and improve development efficiency. The online teaching system based on multi-agent technology provides people with an intelligent online programming method and also injects vitality and energy into online teaching system.

\section{Related technologies}

\subsection{A general review of Agent}

Agent originally means "proxy", that is, a person can be represented by another person. Agent is not a new concept. At present, Agent has been widely used in e-commerce, spacecraft control, and remote education. With application of Agent to these fields, software and system design methods are enriched from a different perspective. What is Agent? What are the distinguishing features of Agent? Agent could be traced back to the book Society of Mind created by Minsky at the earliest. In the book, the writer gave an explanation to Agent and showed individuals exist by relying on society -if there was contradiction between individuals in the process of solving problems, it could be solved through the negotiations or competitions between the individuals that are called as Agent. Since then, professor Minsky further pointed out that these individuals possessed the specific skills in some aspects. According to different research directions and needs, different explanation to Agent is provided. In this paper, Agent is regarded as a concept that provides a new method

This work was supported by the Major Project of National Social Science of China "A Logical, Cognitive and Computational Study on information interaction "(14ZDB016). 
for system design and human observation on the world. Unlike artificial intelligence concepts, human features are not given to it in this studyonly the overall function of it is designed, but the contents of Agent are not discussed. Certainly, its features are always consistent no matter what definition is used for explaining Agent.

\subsubsection{The basic characteristics of Agent}

First, autonomy mainly means that Agent, after being initialized, does not need the intervention from anyone else or other Agents in the runtime, and can adjust its own behaviors and control the internal state according to some methods.

Second, smartness mainly means that it possesses the data, decision-making mechanism, and knowledge demanded for solving relevant problems and implements the functions of screening and recommending the related resources through inference and calculation.

Third, collaboration interactivity can be analyzed from two angles. One angle refers to the Agent's interaction with the users - Agent acquires the user's needs and provides feedback to the user by interacting with the user. The other angle refers to the interaction between Agentsdifferent Agents can mutually transfer information and implement exchange through a communication language, in order to complete the corresponding tasks.

\subsubsection{The classification of Agent}

According to the application of Agent, it is divided into single Agent system, multi-Agent system, and mobile Agent.

First, single Agent system is mainly used for implementing the local tasks and searching information on the web.

Second, multi-Agent system is a system composed of multiple Agents which are in mutual communication and coordination and work together for completing the tasks. It has features such as resource sharing, easy expansion, good reliability, and excellent real-time capability that are also available from an ordinary distributed system; all Agents can solve large-scale complex problems through mutual coordination so that the system can own very powerful robustness, reliability, and self-organizing capacity.

Third, mobile Agent can autonomously move from one host to another host in a heterogeneous online environment, and it is also a type of software entities willing to interact with other Agents, as shown in Fig.1.

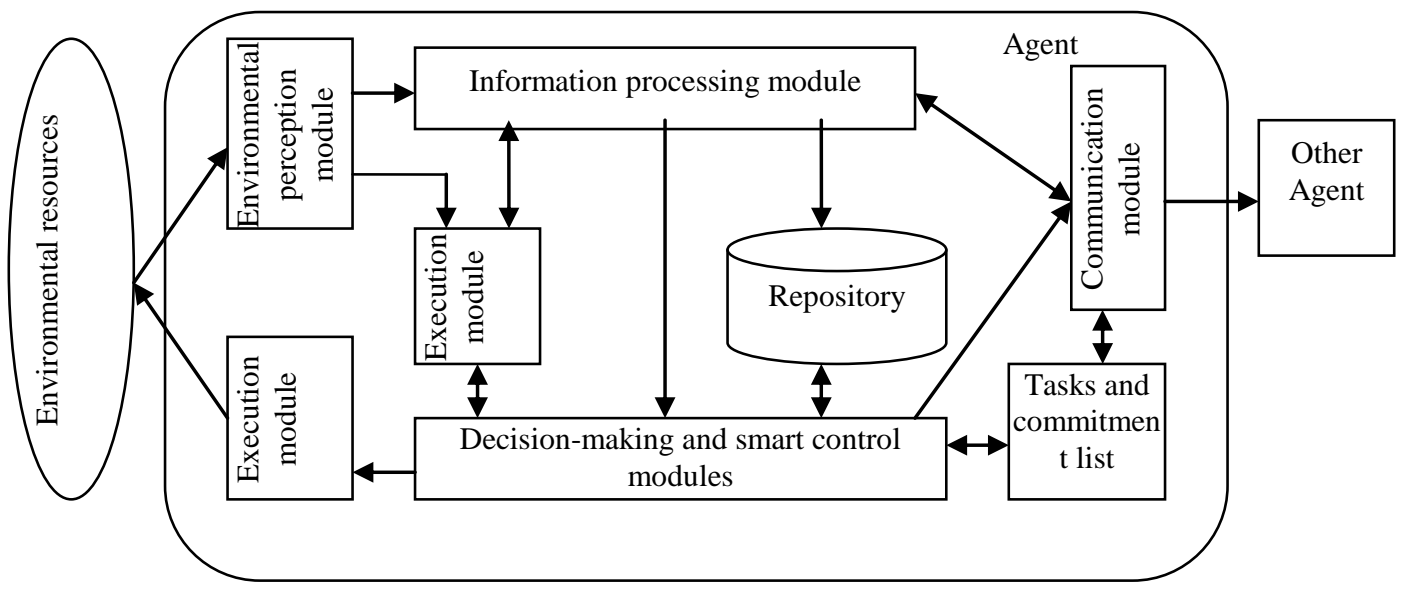

Fig.1 the basic structure of Agent 


\subsection{Multi-Agent system}

Multi-Agent system (MAS) is composed of multiple independent subjects or semi-independent subjects. Multi-Agent system emerges because the ability of a single Agent to solve problems is limited. Multi-Agent system, by relying on communication, promotes a number of Agents to become an integral whole so that the problem-solving ability is greatly improved. The characteristics of Multi-Agent system are concluded as follows.

First, it features sociality. Each Agent is part of Multi-Agent, and a single Agent can communicate with other Agents through a communication language to realize the negotiations between the Agents having different functions and then seek a way for solving problems.

Second, it features autonomy. In Multi-Agent system, other Agents are required to collaborate with each other on the basis of possessing a function providing the demanded service and also desiring to provide such a service, if an Agent sends out a request; an Agent can't implement the enforcement action against another.

Third, it features interoperability. In Multi-Agent system, mutual cooperation, coordination and negotiation are demanded to solve the problems if the goals and functions required by different Agents are different.

\section{The online teaching system design based on Multi-Agent system}

The modeling features of Multi-Agent system are mainly as follows.

First, MAS is a bottom-up, natural and easy-to-expand way of describing nature system, which is used for describing the individual heterogeneity and the complexity of the interaction between different individuals.

Second, existing Agent tools include MASON, Swarm, Repast and Net Logo that can provide a well-improved MAS simulation mechanism and support the implementation of the system simulation of complex system.

Third, how to understand and manage the output results of complex MAS and predict the system development according to the output results remains to be unclear, because it is a kind of "natural" way and has no existing rules or experience to follow. Therefore, classical scientific methods are necessarily used to analyze and forecast the system.

Fourth, it is usually difficult to decompose MAS for getting the local rules of the interaction between individuals under the condition of only knowing the interactive, macro statistical laws between agents.

Fifth, how to make the agent-based model complex properly is very hard to control, but there are no any rules for guiding exactly "what information is necessarily included into the agent-based model". The model has to be properly complex to guarantee (1) the model is simple enough, but can generate the useful knowledge helping understanding the system; (2) the model is complex enough, but can specifically describe the necessary details of the real world.

\subsection{The establishment of the system model}

The application of Agent technology to the online teaching system and is helpful to establish a high level of collaborative online learning environment for unifying the country's education resources under the distributed network environment, further improving the interaction of the network teaching system, and implementing the network intelligent teaching, and creating a different-interface intelligent system based on multiple Agents for different learners. In the online system based on Agent technology, teachers and students are partners who are in a peer relationship and can complete the teaching tasks based on mutual cooperation. The competitive relationship between students is not 
considered temporarily; the cooperation relations are mainly strengthened; the system can provide learning partners for students according to the actual hobbies and knowledge level, and also establish virtual classes for students so that they can communicate with each other and discuss problems together. The overall function of the multi-Agent online teaching system is as shown in Fig.2.

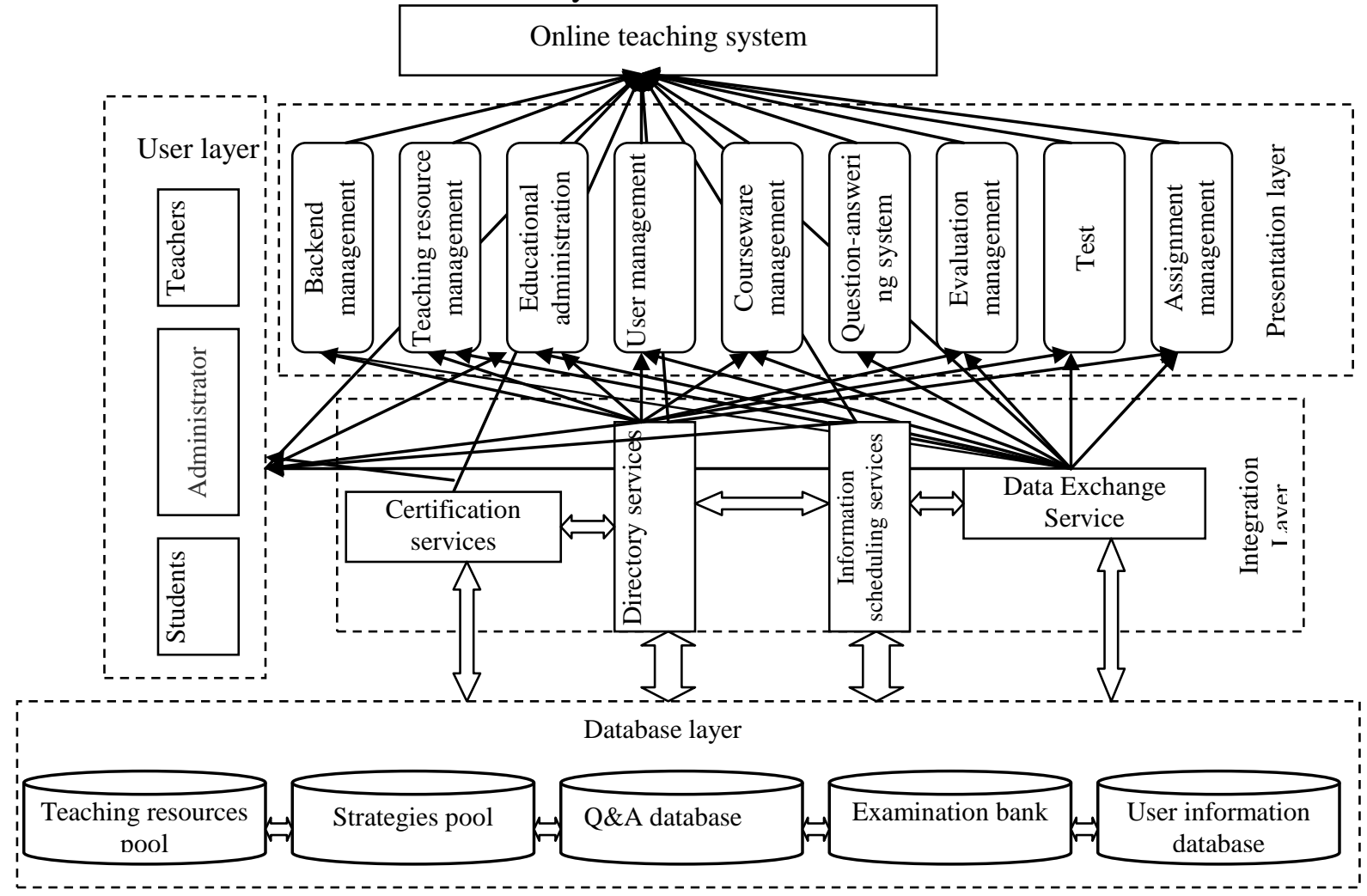

Fig.2 the model of Multi -Agent based online teaching system

\subsection{Specific description to the system}

The database module of this system mainly includes data, teaching resource pool, strategy pool, Q\&A pool, examination question bank, and user information database. The database layer is in the data channel platform layer between users and the operating system and owns the following functions such as data definition, data manipulation, database operation and management, and database establishment and maintenance function and so on. The cooperation between Agents in the multi-Agent online teaching system means that multiple agents work and coordinate together for solving problems and safeguarding the efficiency. It is centered as autonomous Agent and makes the knowledge, desire, plan, purpose, and actions of multiple agents coordinated, for the ultimate goal of achieving consistent collaboration. In the multi-agent based online teaching system, the interaction between Agents is as follows: Agent gets information from other Agents or environment, and then changes self-status and rules based on the obtained information and self-status, and accordingly issues related information to other Agents or environment and also interact with each other.

In the multi-Agent online teaching system, the effective integration of heterogeneous technologies from different disciplines is realized. The system, providing a bright way for coping with this challenge and also being established based on this way, is an online teaching system based on the Agent nodes. As the establishment of multi-Agent online teaching system was raised, 
various heterogeneous smart technologies can continuously get involved in the online teaching system in the form of Agent; the multi-Agent technology accessing to the online teaching system responds to the user's service demand. Practice test proved that many excellent multi-Agent technologies could be continuously applied to online teaching system. Most existing systems always reflect the highest level of group wisdom, and the creativity of group can be understood to be infinite. Therefore, it is necessary to further study the evolution of the smart features of online teaching system.

\section{Conclusion}

In this paper, the present situation of online teaching system is first analyzed, and an online teaching system based on multi-agent technology is established according to the system structure and skills training objective and the modules and functions of the system are defined, aiming to promote the established system to be smarter, more flexible, active and individualized.

\section{ACKNOWLEDGEMENTS}

This work was supported by the Major Project of National Social Science of China "A Logical, Cognitive and Computational Study on information interaction.

\section{References}

[1] Xingtang Li, Bingcheng Liang, Li Liu, Guang-jun He. Complex System Modeling Theory, Method and Technology [M]. Science Press, 2011.

[2] IBM. Web Services for J2EE, Version 1.0. www.ibm.com, 08/2002.

[3] Shu Chen, Binxing Fang, Yonglin Zhou. The Research and Application of P2P Technology [J]. Computer Engineering and Application, 2002, (13).

[4] Xinbin Xu, Jianqing Mi, Lvhua Wang, et al. The Remote Teaching System based on Intelligent Agent [J]. Journal of Zhejiang Shuren University, 2002, 2 (4): 61-63. 